\title{
Anisotropic flow from AGS to LHC energies
}

\author{
P. F. Kolb ${ }^{a}$, J. Sollfrank ${ }^{a}$, U. Heinz ${ }^{b, *}$ \\ ${ }^{a}$ Institut für Theoretische Physik, Universität Regensburg, D-93040 Regensburg, Germany \\ ${ }^{b}$ Theoretical Physics Division, CERN, CH-1211 Geneva 23, Switzerland
}

(March 21, 2018)

Within hydrodynamics we study the effects of the initial spatial anisotropy in non-central heavy-ion collisions on the momentum distributions of the emitted hadrons. We show that the elliptic flow measured at midrapidity in $158 \mathrm{~A} \mathrm{GeV} / c$ $\mathrm{Pb}+\mathrm{Pb}$ collisions can be quantitatively reproduced by hydrodynamic expansion, indicating early thermalization in the collision. We predict the excitation functions of the $2^{\text {nd }}$ and $4^{\text {th }}$ harmonic flow coefficients from AGS to LHC energies and discuss their sensitivity to the quark-hadron phase transition.

The recent observation of transverse collective flow phenomena in non-central heavy-ion collisions at ultrarelativistic beam energies [1] has led to renewed intense theoretical interest in this topic (see [5] for a review). Collective flow is the consequence of pressure in the system and thereby provides access to the equation of state of the hot and dense matter ("fireball") formed in the reaction zone. This access is indirect since the flow in the final state represents a time integral over the pressure history of the fireball. Sorge [6] has argued that different types of transverse flow (radial, directed, elliptic, see [5]) show different sensitivities to the early and late stages of the collision such that a combination of flow observables may allow for a more differential investigation of the equation of state. In particular, he pointed out that the elliptic flow (which develops in non-central collisions predominantly at midrapidity and manifests itself as an elliptic deformation of the hadronic momentum distributions around the beam axis [7] 8 ]) is a signature for the early stage of the collision: its driving force is the spatial eccentricity of the dense nuclear overlap region which, if thermalized quickly enough, leads to an anisotropy of the pressure gradients which cause the expansion. Since the developing anisotropic flow reduces the eccentricity of the fireball, it acts against its own cause and thus shuts itself off after some time. Radial flow, on the other hand, responds to the absolute magnitude of the pressure gradients and not only to their anisotropy; it therefore exists also in central collisions, and in non-central collisions it continues to grow even after the initial elliptic spatial deformation of the fireball has disappeared.

A phase transition from a hadron gas to a colordeconfined quark-gluon plasma causes a softening of the

\footnotetext{
* On leave of absence from Institut für Theoretische Physik, Universität Regensburg. Email: Ulrich.Heinz@cern.ch
}

equation of state: as the temperature crosses the critical value for the phase transition, the energy and entropy densities increase rapidly while the pressure rises slowly. The resulting small ratio of $p / e$ at the upper end of the transition region ("the softest point" [9]) weakens the build-up of flow as the system passes through it. Shuryak [10] and van Hove [11] therefore suggested that a plot of the mean transverse momentum against the central multiplicity density should show a plateau. Later hydrodynamic calculations did not confirm the existence of a plateau, showing only a slight flattening of an otherwise strictly monotonic curve 12]. While the acceleration of the matter is weak in the transition region, the system also takes a long time to cross it, thereby allowing for the flow to build up over a longer time. This considerably reduces the sensitivity of the final radial flow to the existence of a soft region in the equation of state.

Recently van Hove's idea was revived in connection with elliptic flow [13,14]. Sorge [14 used a modified version of RQMD which allows to simulate an equation of state with a "softest point" and found that the response of the elliptic flow to the initial fireball eccentricity was weakened for initial conditions in the phase transition region. Using a hydrodynamic model, Teaney and Shuryak 15 argued that the existence of the phase transition should, at higher energies, also lead to other dramatic effects in the transverse expansion pattern of non-central collisions, in particular to the formation of two well-separated shells moving into the reaction plane. In the present Letter we follow up on these ideas, trying to understand in more detail the transverse dynamics in non-central collisions and what experimental data can tell us about it. We use a similar hydrodynamic approach as in [15,8], adjust its free parameters to data from central $\mathrm{Pb}+\mathrm{Pb}$ collisions at the SPS, demonstrate that it correctly reproduces the measured elliptic flow of pions and protons at midrapidity [2, 17, and then use it to make predictions at other beam energies. In particular we discuss the sensitivity of the excitation functions of $v_{2}$ and $v_{4}$, the $2^{\text {nd }}$ and $4^{\text {th }}$ harmonic flow coefficients, to the existence of a deconfining phase transition.

In the hydrodynamic model one assumes that shortly after the impact the produced strongly interacting matter reaches a state of local thermal equilibrium and subsequently expands adiabatically. In the conservation laws for energy-momentum and baryon number

$$
\partial_{\mu} T^{\mu \nu}(x)=0, \quad \partial_{\mu} j^{\mu}(x)=0
$$


one can then use the ideal fluid decompositions $T^{\mu \nu}=$ $(e+p) u^{\mu} u^{\nu}-g^{\mu \nu} p, j^{\mu}=n u^{\mu}$ in terms of the energy density $e$, the pressure $p$, the (net) baryon number density $n$, and the fluid four-velocity $u^{\mu}$. One thus obtains the equations of ideal (non-dissipative) relativistic hydrodynamics. An equation of state $p(e, n)$ is needed to close the set of equations; its direct connection with the developing flow pattern makes hydrodynamics the most appropriate framework for an investigation of the equation of state.

We are here mostly interested in the transverse expansion dynamics in non-central $(b \neq 0)$ heavy-ion collisions. The lack of azimuthal symmetry leads to a non-trivial 3+1 dimensional problem, requiring considerable numerical resources. As noted in [8], the complexity of the task is significantly reduced if one focusses on the transverse plane at midrapidity and assumes that the longitudinal expansion can be described analytically by Bjorken's scaling solution [18] $v_{z}=z / t$. The latter is known to correctly reproduce the longitudinal expansion dynamics at asymptotically high beam energies, and it works phenomenologically very well even at SPS and AGS energies [19]. This assumption reduces the numerical problem to $2+1$ dimensions. While it should be harmless at midrapidity, it forbids to make reliable predictions at forward and backward rapidities. Hence we cannot describe the rapidity dependence of the transverse flow pattern.

We investigated three different equations of state: (i) an ideal gas of massless particles, $p=\frac{e}{3}$ (EOS I); (ii) a hadron resonance gas including all known resonances 20] with masses below $2 \mathrm{GeV}$ and a repulsive mean field potential $\mathcal{V}(n)=\frac{1}{2} K n^{2}$, with $K=0.45 \mathrm{GeV} \mathrm{fm}^{3}$ 21] (EOS H; for small $n$ this equation of state can be well characterized by the simple relation $p=0.15 e$ ); (iii) an equation of state with a first order phase transition at $T_{\mathrm{c}}(n=0)=164 \mathrm{MeV}$, constructed by matching EOS $\mathrm{H}$ and EOS I using a bag constant $B^{1 / 4}=230 \mathrm{MeV}$ (EOS Q) 21]. EOS Q features at $n=0$ a latent heat of $1.15 \mathrm{GeV} / \mathrm{fm}^{3}$ : the mixed phase ranges from $e_{\mathrm{H}}=0.45$ $\mathrm{GeV} / \mathrm{fm}^{3}$ to $e_{\mathrm{Q}}=1.6 \mathrm{GeV} / \mathrm{fm}^{3}$. We show results only for the semi-realistic cases EOS $\mathrm{H}$ and EOS Q.

For $b \neq 0$ the initial energy density distribution in the transverse plane has an almond shape, characterized by an eccentricity $\epsilon_{x}=\frac{\left\langle\left\langle y^{2}-x^{2}\right\rangle\right\rangle}{\left\langle\left\langle y^{2}+x^{2}\right\rangle\right\rangle}>0$. ( $x$ denotes the transverse direction parallel to the impact parameter $\boldsymbol{b}$, and $\langle\langle\ldots\rangle\rangle$ indicates an energy density weighted spatial average.) This results in larger pressure gradients and thus in larger flow velocities in $x$ than in $y$ direction. Hence the final $p_{\mathrm{T}}$-distribution is anisotropic. Its azimuthal angular dependence can be characterized by (even) Fourier coefficients [16] $v_{2}, v_{4}, \ldots$ (at midrapidity the odd ones, in particular the "directed flow" $v_{1}$, vanish by symmetry):

$$
\begin{aligned}
& \frac{d N}{d \tilde{y} d \varphi}=\frac{d N}{2 \pi d \tilde{y}}\left(1+2 v_{2} \cos (2 \varphi)+2 v_{4} \cos (4 \varphi)+\ldots\right), \\
& \frac{d N}{d \tilde{y} p_{\mathrm{T}} d p_{\mathrm{T}} d \varphi}=\frac{d N}{2 \pi d \tilde{y} p_{\mathrm{T}} d p_{\mathrm{T}}}\left(1+2 v_{2}\left(p_{\mathrm{T}}\right) \cos (2 \varphi)\right.
\end{aligned}
$$

$$
\left.+2 v_{4}\left(p_{\mathrm{T}}\right) \cos (4 \varphi)+\ldots\right)
$$

$\left(\tilde{y}=\operatorname{Artanh}\left(p_{z} / E\right)\right.$ is the longitudinal rapidity of the particles, and $\tilde{y}_{\mathrm{cm}}$ below denotes the midrapidity point.)

For each impact parameter $b$, we parametrize the initial transverse energy density $e(\boldsymbol{r})$ by a Glauber-inspired formula which assumes that the deposited energy is proportional to the number of collisions producing wounded nucleons (for details see 8,22]). The initial baryon number density $n(\boldsymbol{r})$ is taken proportional to $e(\boldsymbol{r})$. The proportionality factors (in particular their $\sqrt{s}$-dependence) cannot be calculated but must be adjusted to data. For adiabatic hydrodynamic expansion there exists a unique relation between the initial entropy density (related to $e_{0}, n_{0}$ by the equation of state) and the final multiplicity density $d N / d \tilde{y}$. We therefore present excitation functions as functions of the (total) pion multiplicity density at midrapidity, where the "energy calibration" $d N_{\pi} /\left.d \tilde{y}\right|_{\tilde{y}=\tilde{y}_{\mathrm{cm}}}(\sqrt{s})$ will be provided by experiment.

The final particle distributions $d N_{i} /\left(d \tilde{y} p_{\mathrm{T}} d p_{\mathrm{T}} d \varphi\right)$ are calculated using the Cooper-Frye formula 23. with a freeze-out hypersurface of constant temperature. We adjust the model parameters, i.e. the initial central energy and baryon densities at $b=0, e_{0}$ and $n_{0}$, the equilibration time $\tau_{0}$, and the decoupling temperature $T_{\mathrm{dec}}$, by fitting 22 the measured 24 negative hadron and proton spectra from central $158 \mathrm{~A} \mathrm{GeV} \mathrm{Pb}+\mathrm{Pb}$ collisions. For EOS Q we find $T_{\mathrm{dec}}=120 \mathrm{MeV}, e_{0}=9.0 \mathrm{GeV} / \mathrm{fm}^{3}, n_{0}=0.95 \mathrm{fm}^{-3}$, and $\tau_{0}=0.8 \mathrm{fm} / c$. The freeze-out temperature and the average radial flow resulting from these initial conditions agree well with previous studies 25 27. In calculating the negative hadron spectrum we included [28] decays of all resonances up to the mass of the $\Delta(1232)$; resonance decays are found to reduce the momentum anisotropies $v_{2,4}$ for pions by $10-15 \%$.

Having adjusted the model parameters in $b=0$ collisions, we can calculate the initial density distributions also for $b \neq 0$ collisions, using the same Glauber formula. The equilibration time $\tau_{0}$ and decoupling temperature $T_{\text {dec }}$ are left unchanged. We have tested this procedure on $p_{\mathrm{T}}$-spectra from non-central $\mathrm{Pb}+\mathrm{Pb}(\mathrm{Au})$ collisions for pions [3] and protons [4 29] and found very good agreement between data and hydrodynamic simulations up to impact parameters of about $10 \mathrm{fm}$ 22. We then proceed to compute the $2^{\text {nd }}$ and $4^{\text {th }}$ harmonic coefficients $v_{2}$ and $v_{4}$ in Eq. (2) as functions of the number of participating nucleons, $N_{\text {part }}$ (or, equivalently, as functions of the impact parameter $b$ ). The results, for EOS $Q$ and the model parameters given above, are shown in Fig. 1.

In addition to $v_{2}=\langle\cos (2 \varphi)\rangle$ (where the average is taken with the particle momentum distribution) we also show the $p_{\mathrm{T}}^{2}$-weighted elliptic flow (denoted as $\bar{\alpha}$ in [8])

$$
v_{2, p_{\mathrm{T}}^{2}}=\frac{\left\langle p_{x}^{2}\right\rangle-\left\langle p_{y}^{2}\right\rangle}{\left\langle p_{x}^{2}\right\rangle+\left\langle p_{y}^{2}\right\rangle}=\frac{\left\langle p_{\mathrm{T}}^{2} \cos (2 \varphi)\right\rangle}{\left\langle p_{\mathrm{T}}^{2}\right\rangle} .
$$

Fig. 1 shows that for pions $v_{2}$ and $v_{2, p_{\mathrm{T}}^{2}}$ differ by an over- 
all factor of about 2, but otherwise have the same impact parameter dependence.

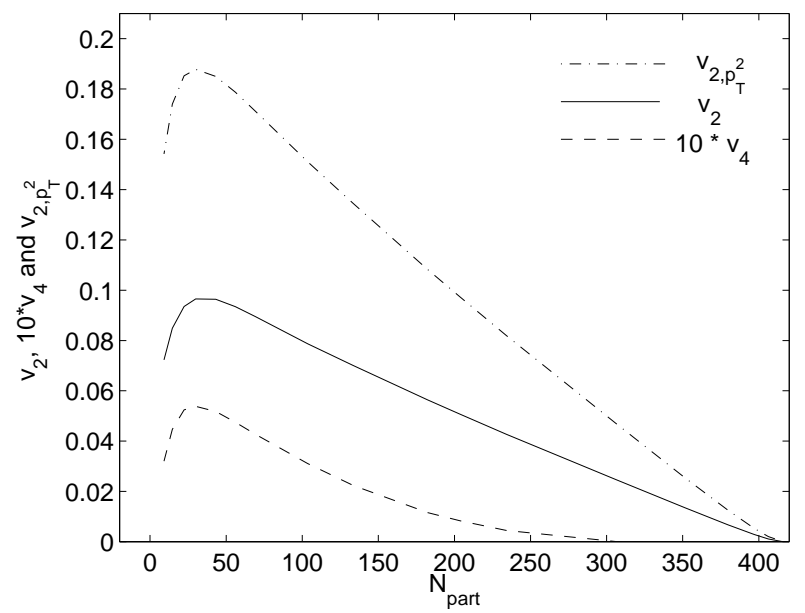

FIG. 1. The $2^{\text {nd }}$ and $4^{\text {th }}$ harmonic flow coefficients for pions as functions of the number of participating nucleons. Also shown is the momentum-space anisotropy $\epsilon_{p}$ (see text).

This factor 2 is important: previously $v_{2}$ and $v_{2, p_{T}^{2}}$ have often been used synonymously and, based on Ollitrault's results [8], one concluded that hydrodynamic calculations overpredict the elliptic flow at the SPS by about a factor of 2. Fig. 2 shows that this is not the case: a correct comparison of the data with the calculated $v_{2}$ (not $v_{2, p_{\mathrm{T}}^{2}}$ !) shows good quantitative agreement. The data 17] were obtained from $\mathrm{Pb}+\mathrm{Pb}$ collisions at the SPS, with a cut on the collision centrality and on the particle $p_{\mathrm{T}}$ as given in the figure. Our calculation was done for $b=7 \mathrm{fm}$ and, using Eq. (3), the same $p_{\mathrm{T}}$-cut as in the data was applied. The resulting values for $v_{2}$ at midrapidity are $2.9 \%$ for pions and $11.7 \%$ for protons. The calculated $p_{\mathrm{T}}$-dependence of $v_{2}$ (not shown) also agrees with the data 22], up to a normalization factor which takes into account that we compute $v_{2}\left(p_{\mathrm{T}}\right)$ at midrapidity while the data [2] were obtained in $4<\tilde{y}<5$ where $v_{2}$ is about a factor 3 smaller (see Fig. 2).

The good agreement of the data (the shape of the $p_{\mathrm{T}^{-}}$ spectra as a function of $b$ and the absolute values and $p_{\mathrm{T}}$-dependences of $v_{2}$ ) with hydrodynamic calculations strongly suggests very early thermalization and pressure buildup in these collisions. In the calculation we can follow the time history of the elliptic flow: we found that $v_{2, p_{\mathrm{T}}^{2}}$ for pions is nearly identical to

$$
\epsilon_{p}=\frac{\left\langle\left\langle T_{x x}-T_{y y}\right\rangle\right\rangle}{\left\langle\left\langle T_{x x}+T_{y y}\right\rangle\right\rangle},
$$

if the spatial average $\langle\langle\ldots\rangle\rangle$ is performed at the time when the fireball center freezes out. $\epsilon_{p}$ is the momentum-space analog of the spatial eccentricity $\epsilon_{x}$ defined above; it does not require knowledge of the particle spectra and can be evaluated also at other times from the solution of the hydrodynamic equations. As expected we find that $\epsilon_{p}$ saturates as soon as the spatial anisotropy $\epsilon_{x}$ goes to zero. For $\mathrm{Pb}+\mathrm{Pb}$ collisions at the SPS, $\frac{1}{6}$ of the final elliptic flow is created while the fireball center is in a pure QGP phase, $\frac{1}{2}$ of it is created in the mixed phase, and the final $\frac{1}{3}$ is generated during the hadronic stage. This agrees with Sorge's conclusion [14 that the elliptic flow at the SPS indeed probes the deconfining phase transition and the existence of a QGP phase.

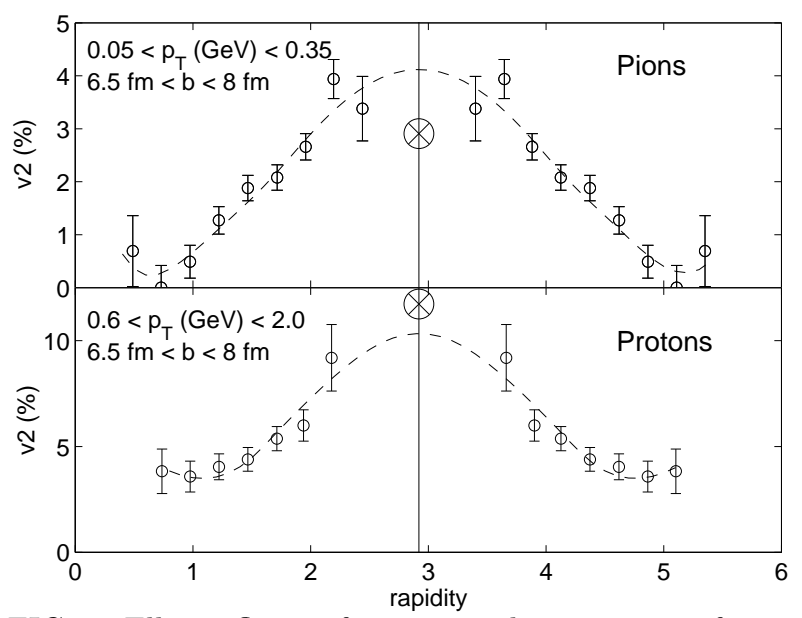

FIG. 2. Elliptic flow $v_{2}$ for pions and protons, as a function of rapidity, as measured by NA49 in $158 A \mathrm{GeV} \mathrm{Pb}+\mathrm{Pb}$ collisions 17]. The dashed lines are to guide the eye. The circled crosses at midrapidity show our hydrodynamic results, with the same cuts in $b$ and $p_{\mathrm{T}}$ as the data.

However, is it also sensitive to the existence of a phase transition? To answer this question we recalculated $v_{2}$ and $v_{4}$ with EOS $\mathrm{I}$ and $\operatorname{EOS} \mathrm{H}$, readjusting the initial conditions to the measured $h^{-}$and $p-\bar{p}$ spectra from central $\mathrm{Pb}+\mathrm{Pb}$ collisions 22]. (While for EOS $\mathrm{H}$ an acceptable fit is possible, the fit for EOS I is quite bad, as found before by several other authors.) Whereas EOS I (which can already be excluded from the $b=0$ spectra) gives about $30-40 \%$ larger values for $v_{2}$, the elliptic flow developed by EOS $\mathrm{H}$ is quite similar to that of EOS Q. $v_{4}$ is about $60 \%$ larger with EOS $\mathrm{H}$ than with EOS Q. The time history of $\epsilon_{p}$ reveals that the softening of the EOS near the phase transition delays the buildup of elliptic flow by about $1.5-2 \mathrm{fm} / c$ but that, at this particular beam energy, in the end it reaches the same value. The mechanism is the same as discussed in the context of van Hove's plateau: the phase transition weakens the elliptic flow, but since the system also spends more time in the transition region, its net effect on $v_{2}$ is much less than naively expected.

Given the apparent insensitivity of elliptic flow to the phase transition at a fixed beam energy, one may still hope for distinctive features in the excitation function of anisotropic flow [30]. In Refs. [13,14] it was suggested that, for initial conditions around the "softest point", the 
response $\frac{\epsilon_{p}}{\epsilon_{x}} \approx 2 \frac{v_{2}^{\pi}}{\epsilon_{x}}$ should develop a plateau-like structure: as the beam energy or the collision centrality is increased and the initial energy density in the fireball center rises from subcritical to supercritical values, one should see a weaker elliptic flow response in the transition region. To check this expectation we calculated excitation functions for $v_{2,4}$ for $\mathrm{Pb}+\mathrm{Pb}$ collisions at fixed impact parameter $b=7 \mathrm{fm}$ (Fig. 3). Since we only varied $e_{0}$ (the initial central energy density in $b=0$ collisions), leaving the other model parameters $\left(\tau_{0}, e_{0} / n_{0}\right.$, and $\left.T_{\mathrm{dec}}\right)$ unchanged, the curves in Fig. 3 correspond to constant initial eccentricity $\epsilon_{x}=0.25$. Hence they give directly the $\sqrt{s}$-dependence (parametrized via $d N /\left.d \tilde{y}\right|_{\tilde{y}_{\mathrm{cm}}}$ ) of the response of the anisotropic flow to $\epsilon_{x}$.

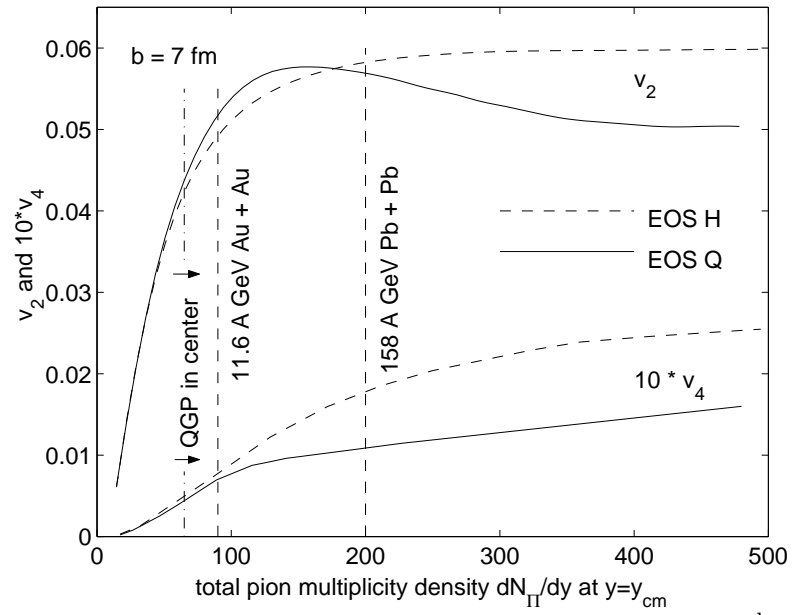

FIG. 3. Hydrodynamic excitation functions of the $2^{\text {nd }}$ and $4^{\text {th }}$ harmonic flow coefficients, $v_{2}$ and $v_{4}$, for pions from $A+A$ collisions $(A \approx 200)$ at impact parameter $b=7 \mathrm{fm}$. The vertical dashed lines indicate the produced total pion multiplicity densities at midrapidity for 11.6 and $158 \mathrm{~A} \mathrm{GeV}$ beam energy (upper ends of the AGS and SPS ranges). The dash-dotted vertical line indicates the threshold above which, at $b=7 \mathrm{fm}$, the fireball center is initially in a pure QGP phase.

Fig. 3 covers the range $1 \leq e_{0} \leq 25 \mathrm{GeV} / \mathrm{fm}^{3}$. Above SPS energies, $v_{2}$ and $v_{4}$ are seen to approach constant asymptotic values. This reflects the saturation of the anisotropic flow before freeze-out and its character as an early stage signature. $v_{2}$ saturates before $v_{4}$ : first the dominant elliptic spatial deformation disappears, the smaller higher-order deformations are washed out later. At lower energies, $v_{2}$ and $v_{4}$ drop due to the decreasing fireball lifetime: the initial central temperature approaches $T_{\mathrm{dec}}$, causing decoupling before the anisotropic flow could fully develop. While this is a generic feature we must caution that quantitatively our results become unreliable in this region: We kept $T_{\text {dec }}$ fixed although at lower beam energies freeze-out is known to occur at lower temperatures [31]; this gives more time for flow buildup, leading to larger values $v_{2}>0$. On the other hand, below 1-2 $A \mathrm{GeV} / c$ the elliptic flow at $\tilde{y}_{\mathrm{cm}}$ builds up before the spectator nucleons have moved out of the way (as we assume); this "inertial confinement" causes the elliptic flow to develop perpendicular to the reaction plane ("squeeze-out" [7], $v_{2}<0$ ) instead of in-plane as in our calculations. Experimentally this sign change of $v_{2}$ occurs near $E_{\text {beam }}=4 A \mathrm{GeV}$ [32]. Neither of these two phenomena is, however, directly related to the existence of a phase transition, and above AGS energies our results are not affected by them.

Fig. 3 shows that the expected weakening of the anisotropic flow due to the phase transition sets in between AGS and SPS energies: comparing the curves for the hadron resonance gas equation of state $(\mathrm{EOS} \mathrm{H}$, dashed) with those for EOS Q (solid), one sees that above SPS energies both $v_{2}$ and $v_{4}$ are reduced for EOS Q. At high energies the anisotropic flow coefficients thus show a qualitatively similar dependence on the EOS as the radial flow [12]. The reduction for $v_{2}$ is relatively small (for pions $v_{2}$ drops from $6 \%$ to $5 \%$ ); $v_{4}$ is reduced by about $40 \%$ but, since $v_{4}$ is so small, this is harder to measure. The effect on $v_{2}$ is also more characteristic, due to the "bump" in $v_{2}$ between AGS and SPS energies resulting from the interplay of the various effects discussed above; for $v_{4}$ the softening of the EOS cannot break the basic monotony of the excitation function.

Within hydrodynamics, the basic phase transition signature in the anisotropic flow is thus a bump (and not the conjectured 13.33 plateau and second rise) in the excitation function of $v_{2}$. Instead of changing $\sqrt{s}$, one can also vary the impact parameter at fixed $\sqrt{s}$ to change the produced multiplicity density $d N_{\pi} /\left.d \tilde{y}\right|_{\tilde{y}=\tilde{y}_{\mathrm{cm}}}$. This provides for an alternate way to study the curves shown in Fig. 3, albeit not at fixed spatial ellipticity $\epsilon_{x}$. We found 22] that in this case the elliptic flow response $\frac{\epsilon_{p}}{\epsilon_{x}} \approx 2 \frac{v_{2}^{\pi}}{\epsilon_{x}}$ develops a similar bump [34]. Unfortunately, for $158 \mathrm{~A}$ $\mathrm{GeV} \mathrm{Pb}+\mathrm{Pb}$ collisions it lies at the limit of the accessible impact parameter range (near $b=11 \mathrm{fm}$ ) where the hydrodynamic approach is expected to break down [35]; for the planned SPS run at $40 \mathrm{AGeV}$, however, the bump should be clearly visible in semicentral $\mathrm{Pb}+\mathrm{Pb}$ collisions.

As a last point we discuss why the seemingly so dramatic phenomenon of the "cracked nut", recently advocated by Teaney and Shuryak [15] as a hydrodynamic signature for the existence of a QGP phase transition, doesn't leave stronger traces in the anisotropic flow pattern. These authors argued that at high energies (RHIC or LHC) a soft region in the EOS leads to the development of a "shell" at the edge of the almond-like initial fireball which is then cracked by the high pressure inside, with two separating half-shells expanding into the reaction plane. While we confirm their numerical results [15], we tend to interpret them more cautiously. To illustrate our point of view we show in Fig. At the freeze-out surface $\tau(x, y)$ for a $\mathrm{Pb}+\mathrm{Pb}$ collision at $b=8 \mathrm{fm}$, initiated with a central temperature $T_{0}=870 \mathrm{MeV}$ at $\tau_{0}=0.2 \mathrm{fm} / c$ and 
yielding a pion midrapidity density $d N_{\pi} /\left.d \tilde{y}\right|_{\tilde{y}=\tilde{y}_{\mathrm{cm}}} \approx 530$ (corresponding to $d N_{\pi} /\left.d \tilde{y}\right|_{\tilde{y}=\tilde{y}_{\mathrm{cm}}} \approx 1600$ for central collisions). Note its mushroom-like structure: While at the SPS hydrodynamics predicts freeze-out surfaces which shrink with time, the present surface features dramatic transverse growth [36] before freezing out nearly instantaneously after $13 \mathrm{fm} / c$. One thus expects a very small emission time duration signal in two-particle BoseEinstein correlations [37], in spite of the phase transition.

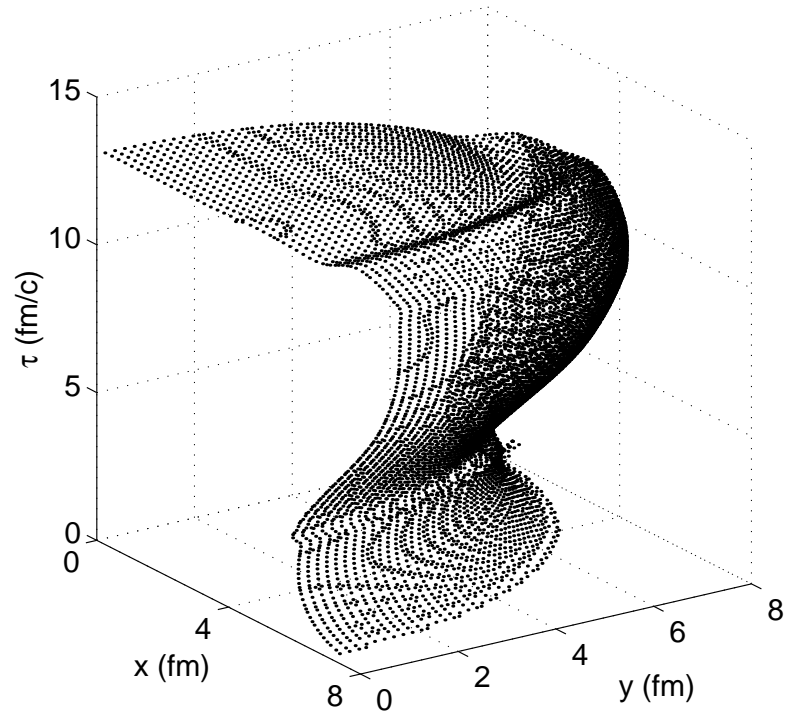

FIG. 4. Freeze-out hypersurface $\tau(x, y, z=0)$ for $b=8 \mathrm{fm}$ $\mathrm{Pb}+\mathrm{Pb}$ collisions, for an initial temperature $T_{0}=1.14 \mathrm{GeV}$ $\left(d N_{\pi} /\left.d \tilde{y}\right|_{\tilde{y}=\tilde{y}_{\mathrm{cm}}} \approx 1600\right)$ in central collisions. Note the dramatic transverse growths, followed by sudden freeze-out.

Already before freeze-out the initial elliptic spatial deformation has vanished. The ripple on the top of the mushroom near its outer edge in $x$-direction is the "nut shell" 15: : in a cut through the surface at $\tau \approx 13 \mathrm{fm} / c$ it shows up as a crescent-shaped half shell at $x \sim 7 \mathrm{fm}$. However, a mere $0.5 \mathrm{fm} / c$ later, the matter in this shell has frozen out, too. For EOS $\mathrm{H}$ one finds a very similar mushroom, but without the ripple at the edge. Since there is no qualitative difference in the momentum-space structure of the "shell" compared to the rest of the matter, this explains why it is impossible to uniquely identify this particular structure by an anisotropic flow analysis. As suggested in [15], two-particle correlations may be more promising, but require extensive studies.

We thank P.V. Ruuskanen, M. Kataja, R. Venugopalan and P. Huovinen for allowing us to use their hydrodynamics code for central collisions and to modify it for the study of non-central collisions. We are indebted to H. Dobler, H. Heiselberg, A. Poskanzer and S. Voloshin for constructive remarks and acknowledge fruitful discussions with E. Shuryak and D. Teaney. This work was supported by BMBF, DFG and GSI.
[1] J. Barrette et al., Phys. Rev. Lett. 73 (1994) 2532.

[2] H. Appelshäuser et al., Phys. Rev. Lett. 80 (1998) 4136.

[3] M.M. Aggarwal et al., Phys. Rev. Lett. 81 (1998) 4087.

[4] F. Ceretto et al., Nucl. Phys. A 638 (1998) 467c.

[5] J.Y. Ollitrault, Nucl. Phys. A 638 (1998) 195c.

[6] H. Sorge, Phys. Lett. B 402 (1997) 251.

[7] H. Stöcker et al., Phys. Rev. C 25 (1982) 1873.

[8] J.Y. Ollitrault, Phys. Rev. D 46 (1992) 229.

[9] C.M. Hung, E.V. Shuryak, Phys. Rev. Lett. 75 (1995) 4003.

[10] E.V. Shuryak, O.V. Zhirov, Sov. J. Nucl. Phys. 28 (1978) 247; Phys. Lett. B 89 (1980) 253.

[11] L. van Hove, Phys. Lett. B 118 (1982) 138.

[12] M. Kataja et al., Phys. Rev. D 34 (1986) 2755.

[13] H. Heiselberg, A.-M. Levy, Phys. Rev. C 59 (1999) 2716.

[14] H. Sorge, Phys. Rev. Lett. 82 (1999) 2048.

[15] D. Teaney, E.V. Shuryak, nucl-th/9904006.

[16] S.A. Voloshin, Y. Zhang, Z. Phys. C 70 (1996) 665.

[17] The data were taken from an update of those published in 21 and can be found at the URL: http://na49info.cern.ch/na49/Archives/Images/Publications/Phys.Rev.Lett.80:4136-4140, 1998

[18] J.D. Bjorken, Phys. Rev. D 27 (1983) 140.

[19] see, e.g., H. Dobler, J. Sollfrank, U. Heinz, nuclth/9904018, Phys. Lett. B, in press; E. Schnedermann, J. Sollfrank, U. Heinz, Phys. Rev. C 48 (1993) 2462.

[20] Particle Data Group, Eur. Phys. J. C 3 (1998) 1.

[21] J. Sollfrank et al., Phys. Rev. C 55 (1997) 392.

[22] P.F. Kolb, J. Sollfrank, U. Heinz, in preparation.

[23] F. Cooper, G. Frye, Phys. Rev. D 10 (1974) 186.

[24] H. Appelshäuser et al., Phys. Rev. Lett. 82 (1999) 2471.

[25] B. Kämpfer et al., J. Phys. G 23 (1997) 2001.

[26] H. Appelshäuser et al., Eur. Phys. J. C 2 (1998) 661.

[27] B. Tomášik, U.A. Wiedemann, U. Heinz, in preparation.

[28] J. Sollfrank, P. Koch, U. Heinz, Z. Phys. C 52 (1991) 593.

[29] P. Braun-Munzinger, J. Stachel, Nucl. Phys. A 638 (1998) 3c.

[30] P. Danielewicz et al., Phys. Rev. Lett. 81 (1998) 2438.

[31] N. Herrmann, Nucl. Phys. A 610 (1996) 49c.

[32] C. Pinkenburg et al. nucl-ex/9903010, Phys. Rev. Lett., in press.

[33] E.V. Shuryak, Quark Matter '99, Nucl. Phys. A, in press.

[34] Our results suggest that the plateau in $\frac{\epsilon_{p}}{\epsilon_{x}}$ found in 14] must be a non-equilibrium effect. Sorge himself stressed 14. that within his approach non-equilibrium mechanisms are responsible for the saturation of $v_{2}$ as a function of time. An experimental study of the excitation functions (Fig. 3) will thus provide valuable evidence for the degree of thermalization reached in the collision, through both their form and magnitude.

[35] Preliminary NA49 data on the $b$-dependence of $v_{2}$ do not show a bump (A. Poskanzer, Quark Matter '99, Nucl. Phys. A, in press).

[36] M. Kataja et al., Z. Phys. C 55 (1992) 153.

[37] U. Heinz, B.V. Jacak, nucl-th/9902020, Ann. Rev. Nucl. Part. Sci. 49 (1999), in press. 